\title{
Análise geoestatística de séries temporais de temperatura do ar, evapotranspiração de referência e precipitação pluvial
}

As variáreis climáticas influenciam diversas atividades do meio urbano e agrícola e o estudo do comportamento dessas variáveis ao longo do espaço e do tempo é fundamental para o planejamento dessas atividades. A geoestatística é uma ferramenta que pode ser usada para modelar e interpolar essas variáveis em escala espacial e temporal. Sendo assim, objetivou-se avaliar o comportamento e a dependência temporal das séries mensais de temperatura média do ar, de evapotranspiração de referência e de precipitação pluvial no período de 2000 a 2020 para a cidade de Montes Claros - MG. Os dados foram obtidos do Instituto Nacional de Meteorologia (INMET). Foi feita uma análise descritiva e semivariográfica das variáveis climáticas e modelos matemáticos foram ajustados aos semivariogramas experimentais. Os dados de temperatura média do ar e de evapotranspiração de referência apresentaram menor variabilidade dos valores quando comparados com os valores de precipitação pluvial. O formato da distribuição dos dados de temperatura média do ar e de evapotranspiração de referência se aproximaram da distribuição normal. A temperatura média do ar e a evapotranspiração de referência apresentaram forte dependência temporal com alcances de 5,6 e 6 meses, respectivamente. A precipitação não apresentou bom ajuste do modelo, conforme constatado na validação cruzada. Contudo, obteve moderada dependência temporal e alcance de 6,3 meses.

Palavras-chave: Geoestatística; Semivariograma; Dependência temporal; Sazonalidade.

\section{Geostatistic analysis of temperature series of air temperature, reference evapotranspiration and pluvial precipitation}

\begin{abstract}
The climatic variables influence several activities in the urban and agricultural environment and the study of the behavior of these variables over space and time is essential for the planning of these activities. Geostatistics is a tool that can be used to model and interpolate these variables on a spatial and temporal scale. Thus, the objective was to evaluate the behavior and temporal dependence of the monthly series of average air temperature, reference evapotranspiration and rainfall in the period from 2000 to 2020 for the city of Montes Claros - MG. The data were obtained from the National Institute of Meteorology (INMET). A descriptive and semivariographic analysis of the climatic variables was made and mathematical models were adjusted to the experimental semivariograms. The average air temperature and reference evapotranspiration data showed less variability of the values compared to the rainfall values. The distribution format of the mean air temperature and reference evapotranspiration data approached the normal distribution. The mean air temperature and the reference evapotranspiration presented strong temporal dependence with ranges of 5.6 and 6 months, respectively. Precipitation did not show a good fit of the model, as found in the crossvalidation. However, he obtained moderate temporal dependence and a range of 6.3 months.
\end{abstract}

Keywords: Geostatistics; Semivariogram; Temporal dependence; Seasonality.

Topic: Tecnologia, Modelagem e Geoprocessamento

Reviewed anonymously in the process of blind peer.
Received: 07/03/2021

Approved: 25/03/2021
Hermes Marçal da Silva (iD)

Universidade Estadual Paulista Júlio de Mesquita Filho, Brasil http://orcid.org/0000-0002-3054-2228

hermesmarcal@hotmail.com

Valéria Cristina Rodrigues Sarnighausen (i)

Universidade Estadual Paulista Júlio de Mesquita Filho, Brasil

http://orcid.org/0000-0003-3943-0211

valeria.sarnighausen@unesp.br
Referencing this:

SILVA, H. M.; SARNIGHAUSEN, V. C. R.. Análise geoestatística de séries temporais de temperatura do ar, evapotranspiração de referência e precipitação pluvial. Revista Ibero Americana de Ciências Ambientais, v.12, n.3, p.710-717, 2021. DOI: http://doi.org/10.6008/CBPC2179$\underline{6858.2021 .003 .0056}$

DOI: 10.6008/CBPC2179-6858.2021.003.0056 


\section{INTRODUÇÃO}

O conhecimento da distribuição espacial e temporal das variáveis climáticas é fundamental para o planejamento das atividades urbanas e agrícolas. No meio agrícola, a produção de alimentos é dependente das condições climáticas. Pereira et al. (2007) salientam a importância do conhecimento da temperatura mensal e da quantidade e distribuição da precipitação pluvial para o planejamento da atividade agrícola. Varejão-Silva (2006) destaca a importância do conhecimento da variabilidade da evapotranspiração para o planejamento e execução da irrigação.

Vários autores estudam o comportamento dessas variáveis climáticas por meio de modelos matemáticos e a análise geoestatística surge como uma ferramenta para modelar e interpolar essas variáveis, tanto em escala espacial quanto em escala temporal. A representação gráfica de fenômenos naturais e sociais é uma forma de abstrair padrões espaciais tornando-se uma ferramenta útil na tomada de decisão (CÂMARA et al., 2004).

A geoestatística é um estudo que leva em consideração, na descrição de um fenômeno, a localização geográfica e a dependência espacial/temporal, introduzindo o conceito de variáveis regionalizadas que são funções numéricas de distribuição espacial/temporal e podem ser expressas por três componentes: a componente estrutural, a componente aleatória e a componente residual (CAMARGO, 1998), relacionadas às tendências, às coordenadas geográficas escolhidas aleatoriamente e aos ruídos, respectivamente.

O uso da geoestatística intensificou-se por ser observada certa correlação/dependência espacial entre dados de fenômenos naturais distribuídos espacialmente (GREGO et al., 2015). Apesar de a análise geoestatística ser amplamente utilizada no âmbito espacial, ainda há poucos estudos no âmbito temporal.

Estudos como estes se fazem importantes em regiões onde há uma distribuição deficitária de chuvas e alto potencial de evapotranspiração, como é o caso de Montes Claros, em Minas Gerais (SOUZA et al., 2018), o que promoverá informações úteis para zoneamento agrícola, épocas mais adequadas de semeaduras, planejamento de irrigação, previsão de geadas, ocorrência de pragas e doenças e classificação climática (ALMEIDA et al., 2011).

Diante disso o objetivo deste trabalho foi avaliar o comportamento e a dependência temporal das séries mensais de temperatura média do ar, de evapotranspiração de referência (ETo) e de precipitação pluvial para a cidade de Montes Claros em Minas Gerais por meio da análise geoestatística, a fim de identificar sazonalidade e tendências.

\section{METODOLOGIA}

Os dados foram obtidos da rede nacional de observações meteorológicas de superfície do Instituto Nacional de Meteorologia (INMET) para a cidade de Montes Claros em Minas Gerais, sendo a estação situada a 645,87 metros de altitude, na latitude $-16,686333$ e longitude $-43,843759$.

A cidade de Montes Claros, inserida na mesorregião Norte de Minas Gerais, se enquadra na classificação climática Aw (clima tropical de savana com estação seca de inverno) de Koppen, caracterizando- 
se por possuir, no período mais frio do ano, temperatura média mensal superior a $18{ }^{\circ} \mathrm{C}$ e precipitação mensal inferior a $60 \mathrm{~mm}$ em pelo menos um dos meses no período de inverno (SÁ JÚNIOR, 2009).

Foram avaliadas as séries mensais de evapotranspiração de referência $(\mathrm{mm})$, de precipitação pluvial (mm) e de temperatura média do $\operatorname{ar}\left({ }^{\circ} \mathrm{C}\right)$ para o período de 2000 a 2020. Foram descartados os meses com ausência de valores.

Uma análise descritiva foi feita com vistas a caracterizar a variabilidade das variáveis durante o período analisado. Sendo assim, determinou-se a média, a mediana, o valor máximo e mínimo, o desvio padrão e os coeficientes de variação, assimetria e curtose. O comportamento dos dados foi verificado com o histograma de frequência e o gráfico de caixa. Essas análises foram realizadas com o software R versão x64 3.6.2 (R CORE TEAM, 2019).

A análise geoestatística foi realizada para avaliar a dependência temporal das variáveis analisadas e determinar os parâmetros dos modelos teóricos de melhor ajuste aos semivariogramas experimentais. 0 semivariograma experimental é uma ferramenta que auxilia na representação quantitativa da variação de uma variável espacialmente distribuída (CAMARGO, 1998) e é definida pela equação 1. Para essas análises foi utilizado o pacote gstat (PEBESMA, 2004; GRALER et al., 2016).

$$
\hat{\mathrm{Y}}(h)=\frac{1}{2 N(h)} \sum_{i=1}^{N(h)}[z(X i)-z(X i+h)]^{2}
$$

Em que: $\hat{Y}(h)$ é o semivariograma estimado, $N(h)$ é o número de pares de valores medidos, $z(X i)$ e $z(X i+h)$ são valores da i-ésima observação da variável regionalizada, Xi um ponto no plano e o h é o vetor distância entre os pontos Xi e $\mathrm{Xi}+\mathrm{h}$, neste caso representando a evolução temporal.

\section{RESULTADOS E DISCUSSÃO}

Os resultados da análise descritiva da temperatura média do ar, da evapotranspiração de referência (ETo) e da precipitação pluvial são apresentados na tabela 1. Os dados climáticos tiveram os valores de média e mediada próximos, com exceção da precipitação pluvial que apresentou o valor da média 2,63 vezes maior que o da mediana, mostrando, portanto, uma distribuição assimétrica entre essas medidas, devido aos valores extremos da média, o que é comum para a variável precipitação, devido a sua sazonalidade. Essa assimetria mostrada na figura 1 torna-se evidente em regiões com a distribuição de chuvas de forma desigual ao longo do tempo, como o observado em Montes Claros (MG), segundo Souza et al. (2018).

Quanto ao coeficiente de variação, os valores de temperatura do ar e de evapotranspiração de referência (ETo) tiveram menor variabilidade dos dados em relação aos dados de precipitação pluvial. A maior variabilidade dos dados de precipitação pluvial pode estar relacionada ao regime pluviométrico irregular, inerente às condições climáticas da região. Collischonn et al. (2008) destacam a elevada aleatoriedade temporal e espacial da precipitação pluvial. 

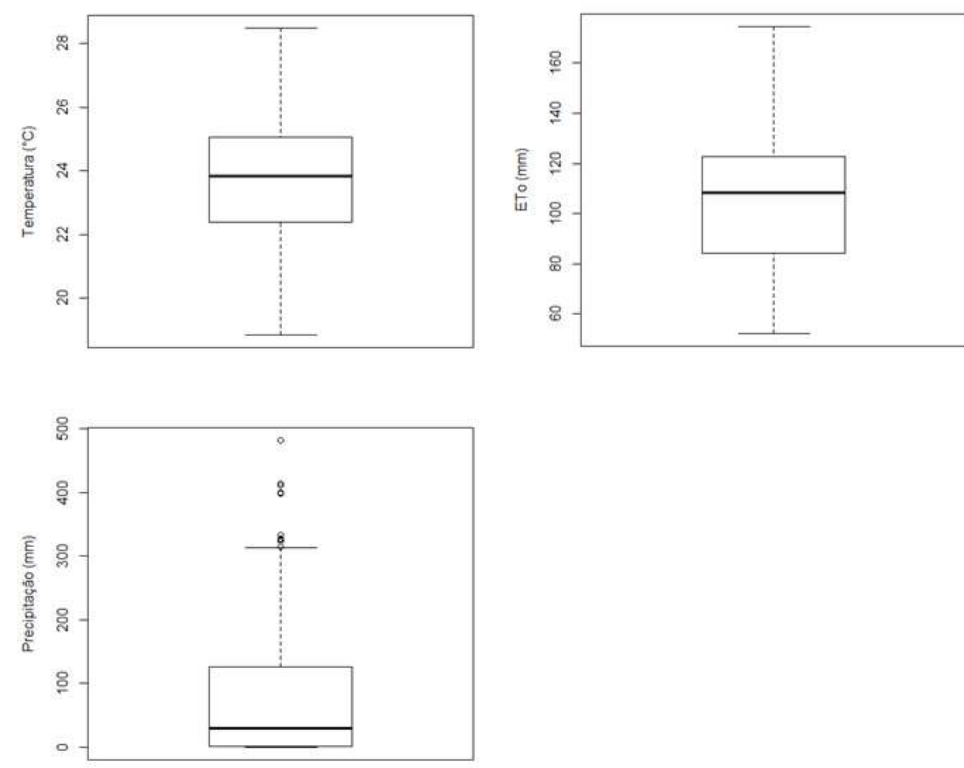

Figura 1: Gráfico de caixa para os dados de temperatura média do ar, evapotranspiração de referência (ETo) e precipitação pluvial.

Tabela 1: Estatística descritiva.

\begin{tabular}{llllllllll}
\hline Variáveis & $\mathrm{n}$ & Média & Mediana & Mínimo & Máximo & $\mathrm{s}$ & $\mathrm{CV}$ & $\mathrm{Cs}$ & $\mathrm{Ck}$ \\
\hline Temperatura $\left({ }^{\circ} \mathrm{C}\right)$ & 237 & 23,71 & 23,84 & 18,85 & 28,5 & 1,9 & 8,01 & $-0,23$ & $-0,29$ \\
ETo (mm/mês) & 188 & 105,94 & 108,49 & 52,28 & 174,5 & 26,61 & 25,12 & 0,16 & $-0,51$ \\
Precipitação (mm/mês) & 239 & 79,94 & 30,4 & 0 & 482,5 & 106,15 & 132,79 & 1,47 & 1,35 \\
\hline
\end{tabular}

n: número de observações; s: desvio padrão; CV: coeficiente de variação (\%); Cs: coeficiente de assimetria; Ck: coeficiente de curtose.

A figura 2 apresenta o histograma de frequência para as três variáveis analisadas. $O$ formato da distribuição dos dados tanto para a temperatura média do ar quanto para a evapotranspiração de referência (ETo) se aproximou da distribuição normal.
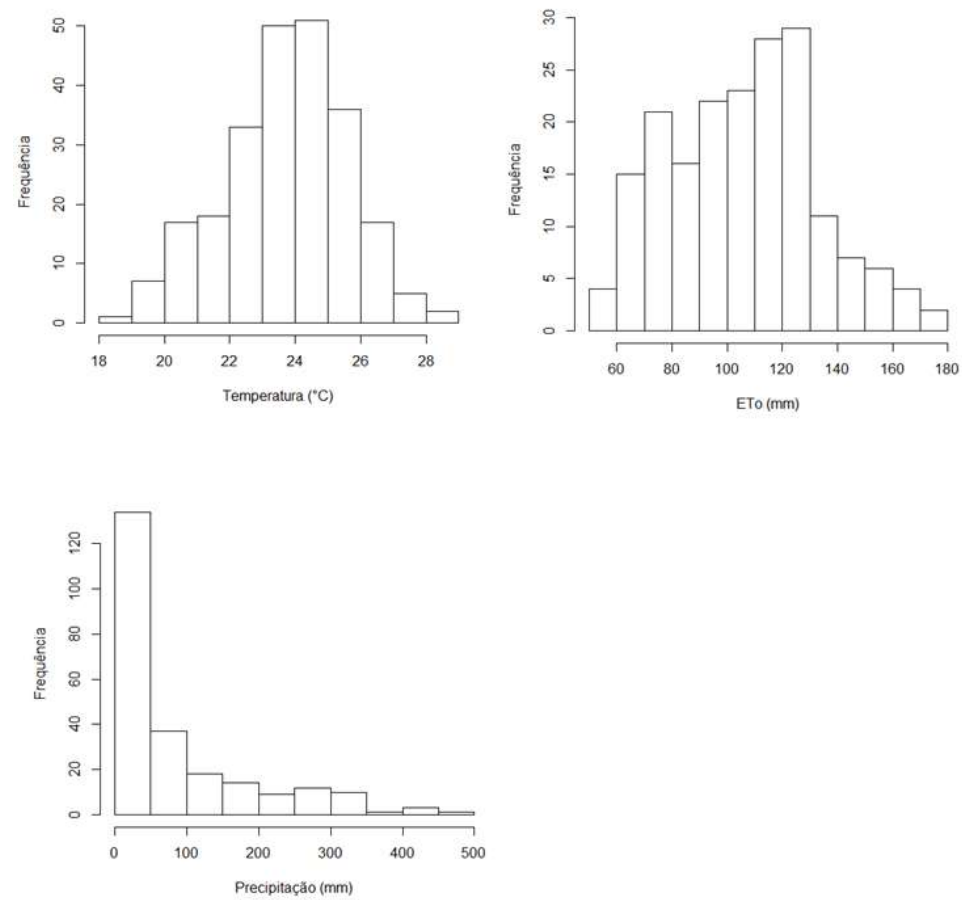

Figura 2: Histograma de frequência dos dados temperatura média mensal, evapotranspiração de referência (ETo) e precipitação pluvial. 
Os valores do coeficiente de assimetria da tabela 1 classificam como distribuição assimétrica negativa $(\mathrm{Cs}<0)$ o formato dos dados de temperatura média do ar, e como assimétrica positiva ( $\mathrm{Cs}>0)$ o formato dos dados de evapotranspiração de referência e precipitação pluvial. Quanto ao achatamento das distribuições, as variáveis se classificaram como platicúrtica $(\mathrm{Ck}<3)$. Isso indica um maior achatamento da distribuição em relação à distribuição normal.

Os mapas dos semivariogramas experimentais estão apresentados na figura 3. É possível notar uma tendência ao longo dos anos representados no eixo x. Ao longo dos meses, eixo y, a tendência se alterna podendo estar associado às estações do ano.
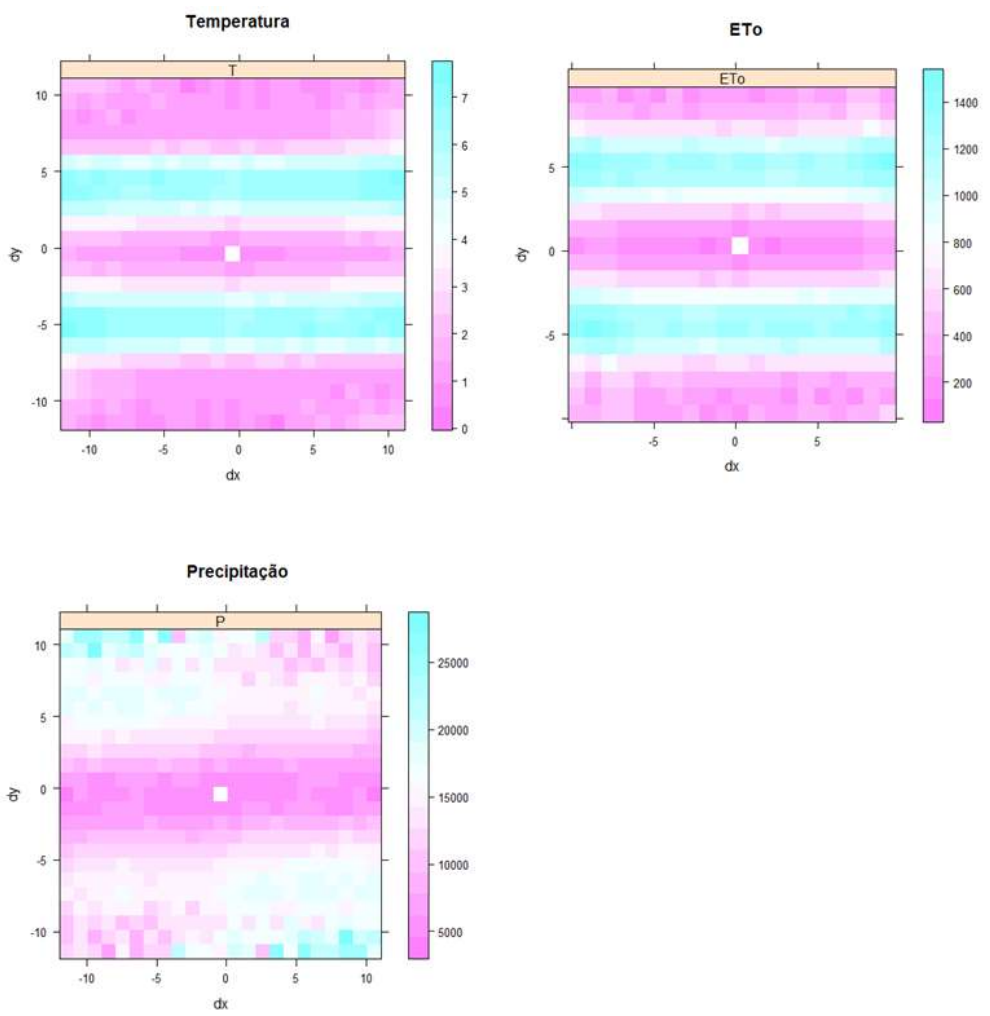

Figura 3: Mapas dos semivariogramas experimentais para os dados de temperatura média mensal, evapotranspiração de referência (ETo) e precipitação pluvial.

A tabela 2 apresenta os parâmetros dos modelos teóricos ajustados aos semivariogramas experimentais. Conforme a classificação proposta por Zimback (2001) as variáveis temperatura e evapotranspiração de referência (ETo) se enquadraram na classe forte de dependência temporal (IDT $\geq 75 \%)$, já a precipitação se enquadrou na classe moderada de dependência temporal ( $25 \% \leq$ IDT $\leq 75 \%)$. Essas afirmações retratam o que é verificado na figura 3, em que é possível identificar a sazonalidade, ou tendência, mais definida para a temperatura e evapotranspiração quando comparadas com a precipitação.

Sartori et al. (2010) obtiveram forte dependência temporal para dados de temperatura (99) e precipitação (80) para a cidade de Botucatu - SP, à qual apresenta sazonalidade melhor definida quando comparada com a cidade do presente estudo.

A figura 4 ilustra os semivariogramas experimentais e a curva de ajuste. O modelo esférico foi o modelo teórico de melhor ajuste aos dados de temperatura e evapotranspiração de referência (ETo). O mesmo foi encontrado por Ramos et al. (2009) que obteve melhor ajuste do modelo esférico aos dados de 
temperatura com $R^{2}$ e IDT de 0,986 e 0,96 respectivamente para o ano de 2001 , e de 0,993 e 0,95 respectivamente para o ano de 2002.

Tabela 2: Parâmetros dos modelos ajustados aos semivariogramas das variáveis climáticas.

\begin{tabular}{llllllll}
\hline Variáveis & Modelo & $\mathrm{A}_{0}$ & $\mathrm{C}_{0}$ & $\mathrm{C}_{0}+\mathrm{C}_{1}$ & $\mathrm{R}^{2}$ & $\mathrm{RMSE}$ & IDT \\
\hline Temperatura $\left({ }^{\circ} \mathrm{C}\right)$ & Esférico & 5,6 & 1 & 4,2 & 0,74 & 0,97 & 0,76 \\
ETo (mm/mês) & Esférico & 6 & 190 & 800 & 0,76 & 12,90 & 0,76 \\
Precipitação (mm/mês) & Gaussiano & 6,3 & 6000 & 12000 & 0,43 & 79,85 & 0,50 \\
\hline
\end{tabular}

$A_{0}$ : alcance da dependência temporal (meses); $C_{0}$ : efeito pepita; $C_{0}+C_{1}$ : patamar; $R^{2}$ : coeficiente de determinação; RMSE: raiz quadrada do erro médio; IDT: índice de dependência temporal (\%).

O modelo Gaussiano, apesar de ter sido o melhor modelo para os dados de precipitação pluvial, apresentou $\mathrm{R}^{2}$ de 0,43 e RMSE de 79,85. Almeida et al. (2011) encontrou valores semelhantes utilizando metodologia geoestatística para modelar séries temporais de precipitação, obtendo $R^{2}$ de 0,40 e RMSE de 119.
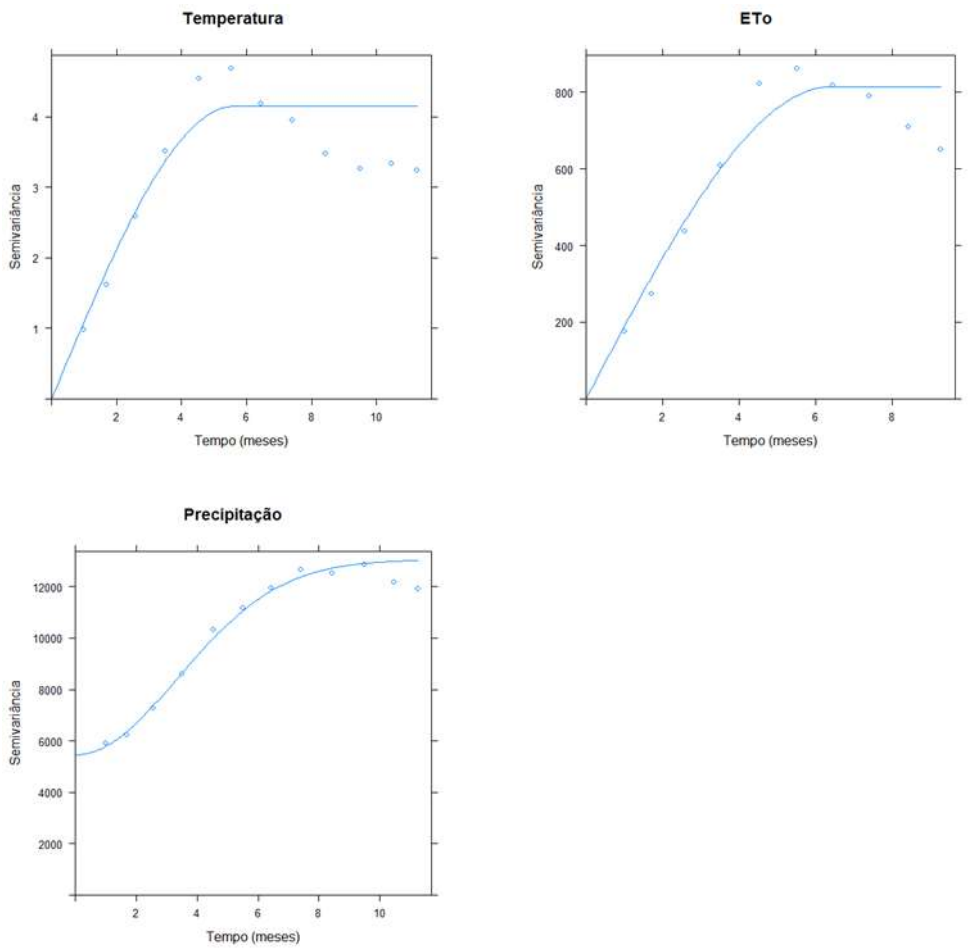

Figura 4: Semivariograma da temperatura, da evapotranspiração de referência (ETo) e precipitação pluvial.

As variáveis climáticas apresentaram dependência temporal, contudo a validação cruzada, figura 5, ilustra o menor desempenho do modelo teórico, ajustado ao semivariograma experimental, para predizer valores de precipitação pluvial, corroborando com WILLIAMS et al. (2009) que não obtiveram boa descrição de dados de precipitação pluvial por técnicas geoestatísticas. 

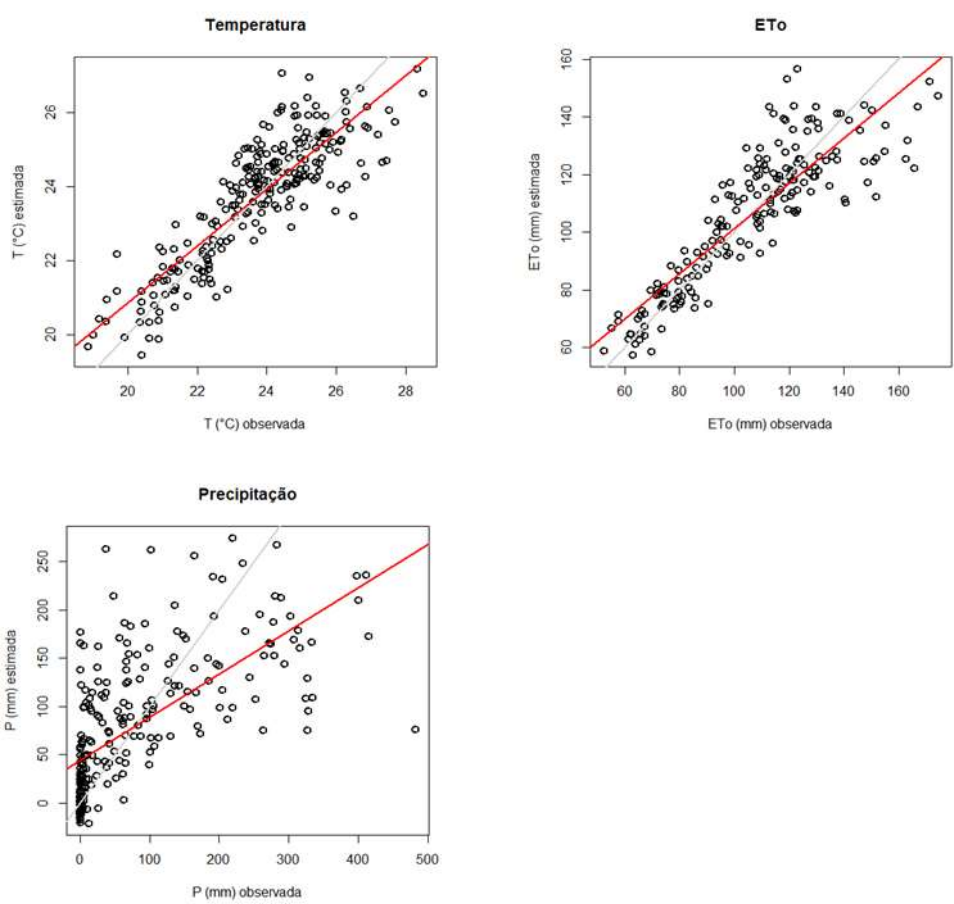

Figura 5: Validação cruzada entre os dados observados e estimados pelo modelo de semivariograma das variáveis temperatura, evapotranspiração de referência (ETo) e precipitação.

\section{CONCLUSÕES}

As séries mensais de temperatura do ar e evapotranspiração de referência apresentaram forte dependência temporal, o que mostram os valores de $R^{2}$ e RMSE para os valores estimados na validação cruzada, quando comparados aos valores para as séries mensais de precipitação.

A série mensal de precipitação pluvial apresentou moderada dependência temporal, elevada variabilidade temporal e obteve baixa descrição dos valores estimados na validação cruzada.

\section{REFERÊNCIAS}

ALMEIDA, A. Q.; RIBEIRO, A.; PAIVA, Y. G.; RASCON, N. J. L.; LIMA, E. P.. Geoestatística no estudo de modelagem temporal da precipitação. Revista Brasileira de Engenharia Agrícola e Ambiental, Campina Grande, v.15, n.4, p.354-358, 2011.

CÂMARA, G.; MONTEIRO, A. M. V.; DRUCK, S.; CARVALHO, M. S.. Análise espacial e geoprocessamento. In: DRUCK, S.; CARVALHO, M. S.; CÂMARA, G.; MONTEIRO, A. M. V.. Análise espacial de dados geográficos. Brasília: Embrapa, 2004.

CAMARGO, E. C. G.. Geoestatística: fundamentos e aplicações. In: CÂMARA, G.; MEDEIROS, J. S..

Geoprocessamento para projetos ambientais. 2 ed. São José dos Campos: INPE, 1998.

COLLISCHONN, W.; TASSI, R.. Introduzindo hidrologia. Porto Alegre: IPH UFRGS, 2008.

GRALER, B.; PEBESMA, E.; HEUVELINK, G.. Spatio-temporal interpolation using gstat. The R Journal 8, v.1, p.204-218, 2016.

GREGO, C. R.; OLIVEIRA, R. P. O.. Conceitos básicos da geoestatística. In: OLIVEIRA, R. P.; GREGO, C. R.; BRANDÃO, Z. N.. Geoestatística aplicada na agricultura de precisão utilizando o vesper. Brasília: Embrapa, 2015. p.41-62.

PEBESMA, E. J.. Multivariable geostatistics in S: the gstat package. Computers e Geosciences, v.30, p.683-691, 2004.

PEREIRA, A. R.; ANGELOCCI, L. R.; SENTELHAS, P. C. Meteorologia agrícola. Piracicaba: Universidade de São Paulo, 2007.

R CORE TEAM. R: A language and environment for statistica computing. Vienna: R Foundation for Statistical Computing, 2019.

RAMOS, C. M. C.; NARDINI, R. C.; ZIMBACK, C. R. L.; SERAPHIN, S.. Análise temporal da variação da temperatura utilizando-se a geoestatística. In: SIMPÓSIO BRASILEIRO DE SENSORIAMENTO REMOTO, 14. Anais. Natal: INPE, 2009.

SÁ JÚNIOR, A.. Aplicação da classificação de Koppen para o zoneamento climático do estado de Minas Gerais. Dissertação (Mestrado em Engenharia Agrícola/Engenharia de Água e Solo) - Universidade Federal de Lavras, Lavras, 2009. 
SARTORI, A. A. C.; SILVA, A. F.; RAMOS, C. M. C.; ZIMBACK, C. R. L.. Variabilidade temporal e mapeamento dos dados climáticos de Botucatu-SP. Irriga, v.15, n.2, p.131-139, 2010.

SOUZA, D. O.; OLIVEIRA, F. G.; CASTRO, I. L.; SOARES, J. B. S.; REIS, M. M.; FIGUEIREDO, F. P.. Frequência de ocorrência de precipitação pluviométrica em Montes Claros-MG. Revista Agrarian, v.11, n.42, p.337-342, 2018.

VAREJÃO-SILVA, M. A.. Meteorologia e climatologia. Recife: versão digital 2, 2006.
WILLIAMS, M.; SPADAVECCHIA, L.. Can spatio-temporal geostatistical methods improve high resolution regionalisation of meteorological variables?. Agricultural and Forest Meteorology, v.149, p.1105-1117, 2009. DOI: http://doi.org/10.1016/j.agrformet.2009.01.008

ZIMBACK, C. R. L.. Análise espacial de atributos químicos de solos para fins de mapeamento da fertilidade. Tese (Doutorado em Levantamento do solo e fotopedologia) Universidade Estadual Paulista "Júlio de Mesquita Filho", Botucatu, 2001.

A CBPC - Companhia Brasileira de Produção Científica (CNPJ: 11.221.422/0001-03) detém os direitos materiais desta publicação. Os direitos referem-se à publicação do trabalho em qualquer parte do mundo, incluindo os direitos às renovações, expansões e disseminações da contribuição, bem como outros direitos subsidiários. Todos os trabalhos publicados eletronicamente poderão posteriormente ser publicados em coletâneas impressas sob coordenação da Sustenere Publishing, da Companhia Brasileira de Produção Científica e seus parceiros autorizados. Os (as) autores (as) preservam os direitos autorais, mas não têm permissão para a publicação da contribuição em outro meio, impresso ou digital, em português ou em tradução. 